\title{
Research Progress of Case Management and Evaluation of Quality of Life in Patients with Diabetes by Out-Patient Nurses
}

\author{
Liyun Chen', Caixue Long1, Chenyun Xu' ${ }^{1}$ Jun Zheng ${ }^{2}$ \\ ${ }^{1}$ Hainan General Hospital, Haikou, China \\ ${ }^{2}$ The Fourth People's Hospital of Haikou, Haikou, China \\ Email: g2002m@163.com
}

How to cite this paper: Chen, L.Y., Long, C.X., Xu, C.Y. and Zheng, J. (2020) Research Progress of Case Management and Evaluation of Quality of Life in Patients with Diabetes by Out-Patient Nurses. Journal of Diabetes Mellitus, 10, 245-254. https://doi.org/10.4236/jdm.2020.104020

Received: October 26, 2020

Accepted: November 21, 2020

Published: November 24, 2020

Copyright $\odot 2020$ by author(s) and Scientific Research Publishing Inc. This work is licensed under the Creative Commons Attribution International License (CC BY 4.0).

http://creativecommons.org/licenses/by/4.0/

\begin{abstract}
Diabetes is the world's third largest threat to people's health. In China, the incidence of diabetes is on the rise, the cure rate of diabetes is low, patients with complications are more, and diabetes patients bring a heavy economic burden to the family and society. Compared with developed countries, China still has a big gap in terms of diabetes care, education and management, and even less long-term follow-up of diabetes education and management results. Therefore, the management of diabetic patients should not only stay in the intervention during hospitalization, but also extend to the outside of the hospital. Only by implementing the whole-process nursing case management integrating hospitalization-family/community-outpatient, can diabetic patients better control blood glucose, delay or prevent the occurrence of chronic complications, and improve their quality of life. The nursing work method of case management is adopted to carry out personalized assessment for diabetes patients, design the process of initial outpatient consultation, content and process of follow-up consultation for nurses, and implement personalized guidance. The diabetic complications should be screened regularly, diabetes management should be standardized, self-care ability and behavior compliance of treatment and nursing of diabetic patients should be improved, and comprehensive whole-process nursing management should be provided for every diabetic patient, so as to improve the quality of life of patients and truly benefit diabetic patients.
\end{abstract}

\section{Keywords}

Diabetes, Outpatient Case Management, Questionnaire Survey, The Quality of Life 


\section{Introduction}

Diabetes Mellitus (DM) is a chronic lifelong disease. Advanced complications are a major threat to people's physical and mental health. It is widely recognized as the three major killers of human life together with coronary heart disease and cancer [1]. According to a report by the World Health Organization (WHO), in 2007, there were 189 million confirmed cases of diabetes worldwide, and it is predicted that the number will reach 300 million in 2025. At present, there are nearly 150 million patients with diabetes in China, and nearly 100 million people are undiagnosed diabetic patients. Diabetes has become the fourth non-communicable chronic disease in China [2]. China has about 1 million new cases of diabetes every year. The situation is serious. Among the elderly with high morbidity, diabetic patients account for about $95 \%$, while the incidence of long course of disease, diabetic patients, coronary heart disease, ischemic disease, insomnia, and limb necrosis is significantly higher than that of the diabetic group with severe complications. Obviously, diabetes has become a universal public health problem that seriously threatens the health of the Chinese people [3]. In recent years, the prevention and treatment of diabetes in China has gradually attracted extensive attention from patients, their families, medical personnel and the society. Adopt various methods and measures to improve the ability of diabetic patients to self-manage diabetes and reduce their dependence on medical institutions. However, the majority of Chinese (especially) elderly people with diabetes have little or no knowledge of the basic knowledge, treatment and self-care of diabetes. Due to their lack of knowledge and understanding, they fail to follow the prevention and treatment of diabetes, which often leads to poor blood sugar control and chronic complications of diabetes [4]. On the other hand, since the treatment of diabetic patients requires life-long diet control, appropriate exercise, regular blood glucose monitoring and other lifestyle and comprehensive management of treatment methods [5], in the absence of professional supervision and guidance, diabetic patients strictly follow medical institutions. The medical guidance, scientific treatment and self-management provided by DM will provide good treatment effects for diabetic patients. Therefore, the intervention of inpatients alone is not enough. Professional nursing intervention should be extended to patients' families and outpatient clinics in hospitals. Patients with diabetes history should realize the whole process of nursing intervention in families and outpatient clinics in hospitals, so as to help patients with diabetes improve their understanding of diabetes knowledge, improve their medical compliance and good blood sugar control, and truly improve patients' quality of life [6].

\section{Case Management of Diabetic Patients}

\subsection{Generation of Whole-Process Nursing Management}

The entire Management Case Management (CM) was originally proposed by the United States, it can be traced back to 1860, the causes of it was the poor and in- 
creasing, but the lack of corresponding health care system to regulate and guarantee the health of these people, a century later in 1960, the entire Management formal formation of the word, the cause of the formation is to adjust the contradictions, at a time when this kind of contradiction is becoming more complex [7]. In the late 1980s, in order to solve the problem of rising medical costs, the United States adopted effective measures through whole-process management, which not only reduced medical costs, but also ensured that patients could get the medical services they needed in the whole medical process [8]. Whole-process management is a collaborative process that includes assessment, planning, execution, coordination, monitoring and evaluation to select medical services [9], to meet the health needs of patients, and to apply communication and available treatment conditions to achieve high-quality and reasonable results. Whole-process management has also become a mechanism of service coordination, which is used to simplify the procedures for special groups such as frail elderly people to transfer to hospital or community [10]. In most of the whole management system, the nurse acts as a management responsibility, in the 21 st century, the entire management has many is completed by the Nurse in the whole process of the Nurse care management (Nurse Case Manager sliding), the Nurse's role is to partner, combination, coordinator, they make sure with the least amount of resources, to meet the medical needs of patients [11] and purpose. According to WHO, from 1995 to 2025, the growth rate of diabetes in China will reach 68\%, ranking the first in the world, and the absolute number of diabetes patients will reach 50 million [12].

Along with the rising prevalence of obesity and the improvement of people's understanding of the disease, and diabetes prevention and control work has been more and more be taken seriously, but compared with developed countries, China in diabetes full management still exist some problems to be solved: first, is not regulated by the administrative department of public health community management mode or management norms, community management does not reach the designated position; Secondly, many urban and rural community patients do not have corresponding hospital jurisdiction, community patients are in the state of no management; Third, the hospital management of patients in the whole process of the implementation of part of bureau be confined to the hospital, most of the hospital, has been conducted in the fourth, hospital and community lack of cohesion, the vast majority of patients discharged from hospital didn't pass the hospital and community cohesion, they didn't get after discharge professionals should have the continuity of the specialized nursing instruction, patients discharged from hospital after rely on consciously to self-management, so often make patients with early complications, accelerate the next time [13] in hospital.

\subsection{Significance of Setting Up a Specialist Nurse Clinic for Case Management of Diabetic Patients}

In 2004, WHO pointed out that in 2002, chronic non-communicable diseases represented by cardiovascular diseases, diabetes, obesity, cancer and respiratory 
diseases accounted for $58.8 \%$ of the total number of deaths and $46.8 \%$ of the global burden of disease, which is expected to rise to $73 \%$ and $60 \%$ respectively by 2020 [14]. At present, China's hospital is the main place for disease prevention and cure basically, but ill go to a doctor is no longer the sole purpose of people to the hospital, they not only to see the doctor, do all kinds of examination, to buy medicine, more is want to obtain the required health knowledge, solve doubts, help them establish healthy behavior, issued the pure medicine services have not satisfied [15]. Developing specialist nurse clinics could make up due to the outpatient doctor accepts patients more daily, busy, no time for all kinds of questions put forward by the patients in detail, through the examination on patients with a full range of lifestyle, self management, such as guidance, play the advantages of diabetes specialist nurses, conduct work for the secondary prevention of diabetes and to reduce the morbidity and mortality of the disease, to improve the health quality of the patients has a positive function and meaning.

\subsection{The Role of Nurses in Case Management of Patients with Diabetes}

Clinical Nurse Specialists (CNS) are also referred to as Clinical nursing experts, who have profound theoretical knowledge, rich Clinical experience and excellent Clinical skills in a certain field of Clinical nursing, and directly provide high-quality nursing services to patients [16]. Diabetes specialist nurses are nursing specialization in health nurses in the process of formation of a division of labor, CNS is a senior nurse, specialist nurse training in developed countries has developed to a mature, diabetes specialist nurses in the life of patients with diabetes health care plays an important role, is the bridge of diabetes patients and community health care and even doctors, is melts the health education, health guidance, nursing care, and even the medical personnel of social work is an organic whole, it has become more and more get the role of nurses, doctors and social identity. With the transformation of medical model and the diversification of social needs, there is an urgent need to be convenient and prevention, treatment and nursing, rehabilitation, social support, psychological care and health services and guidance, the existing primary care doctors and clinicians cannot meet the increasing health needs, the nurse clinic (nurse. 1 CD's a-clinic, NLC) arises at the historic moment.

NLC was developed earlier in foreign countries, and is generally composed of several CNS and clinical medical consultants of corresponding disciplines, and consists of nurs-led teams, which are divided into different specialized outpatient clinics, such as orthopedic outpatient clinic, radio-chemotherapy outpatient clinic, fistula outpatient clinic, pre-operation evaluation outpatient clinic, pressure ulcer outpatient clinic, etc. [17]-[22]. Since 2002, Guangdong Provincial People's Hospital of China has been the first to set up a specialized nurse clinic for stomatology in the province. In 2004, it opened a specialized nurse clinic for diabetes. In 2006, another specialized nurse of diabetes also set up a specialized 
nurse clinic for diabetes, providing specialized and diversified health guidance for diabetes patients. In the past few years, the specialist nurse clinic has become more and more influential. Doctors, nurses or patients will refer patients to the specialist nurse clinic, and the diabetes specialist nurse clinic is playing an increasingly important role.

\section{Effect Evaluation of Quality of Life in Diabetic Patients}

\subsection{Effect Evaluation Tools for Quality of Life in Diabetic Patients}

1) Questionnaire for general information of patients: including age, sex, residence status, and frequency of visits of children, etc.

2) Diabetes self-management Activity Scale: The self-management behavior scale designed by Toobert et al. [23] was developed for diabetic patients over 60 years old. The scale includes 13 items in 6 dimensions, including diet management, exercise management, medication on doctor's advice, self-glucose monitoring, foot care and smoking status. Among them, the smoking dimension statistics the number of smokers and the daily smoking amount, and does not calculate the score. The scores of other dimensions range from 0 to 7 points. The questionnaire was scored in each dimension separately, and the higher the score was, the better the self-management behavior was. After the scale was sinicized, the content validity coefficient (CVI) assessed by experts was 1.00 [24], and Cronbach's coefficient of each dimension was detected between 0.74 and 0.88 .

3) Diabetes Specific Quality of Life (DSQL) scale [25]: Designed and developed by Zhou Fengqiong et al., the Quality of Life of diabetic patients was evaluated. DSQL scale, a total of 27 items, including four dimensions, respectively is physiological function (12 items), mental/spiritual (8 items), social relations (4 items), treatment (3 items), each item according to the "no" "occasional" "(about half the time)" "often" "there is always" give 1 - 5 points, respectively, the sum of the entries for the total value. The lower the overall score, the better the quality of life. The contribution of all factors: physiological $50.0 \%$, psychological $3.5 \%$, social relations $4.4 \%$, treatment $16.7 \%$, a total of $74.6 \%$, indicating that the scale has good structural validity [26]. Cronbach's coefficient of the total scale measured by the experiment was 0.95 .

\subsection{Intervention Methods of Quality of Life Nursing for Diabetic Patients}

1) Cognitive intervention: each patient's living environment, education and personal experience are not the same, there will be some differences in the understanding of diabetes, cognitive ability and the degree of communication difficulty are also different. Therefore, according to the actual situation of patients, the treatment methods, prevention methods, inducing factors, clinical symptoms and clinical treatment progress of diabetes should be explained to patients. For the misunderstanding of patients, targeted guidance and emphasis should be given to enable patients to grasp the knowledge related to diabetes [27]. For 
some patients with poor reception ability and older age, one-to-one intervention should be adopted to provide health guidance to patients, and let patients' family members participate in the nursing process, give patients care and care, encourage patients, so that patients develop healthy living habits.

2) Psychological intervention: diabetes is a chronic disease that requires long-term treatment, with a long course of disease and a large fluctuation range of blood sugar level. In addition, the elderly have a low psychological tolerance, which is prone to fear, anxiety and tension, causing adverse effects on the treatment of the disease and the quality of life. Therefore, psychological intervention should be carried out to help patients maintain a healthy attitude and improve their negative emotions [28]. Patients' psychological pressure can be relieved through psychological adjustment and psychological counseling, and attention, confidence rebuilding and relaxation therapy can be adopted to let patients relax their body and mind. Communicate actively with the patient, kind, gentle and friendly attitude towards patients, give patients with respect and understanding, establish good relationship with the patient, answer the patient's doubt, reduce the patient's psychological burden, correcting the bad mood, and guide patients to bear myself, in a timely manner to eliminate negative emotions, help build up my confidence in the treatment of patients, improve the compliance of patient care. In terms of patients' daily life and living, medication, activities, daily diet, etc., care and nursing should be given. For patients with serious illness who need long-term treatment, active communication and psychological counseling should be conducted so that patients can maintain a positive attitude towards treatment.

3) Exercise intervention: the right amount of exercise can effectively improve the body resistance of patients, is conducive to health. Patients should be guided to exercise according to their actual conditions, and the appropriate exercise mode should be selected according to the contraindications and indications of patients [29]. You can choose jogging, tai chi, aerobics, walking and other sports, pay attention to grasp the amount of exercise, to avoid excessive fatigue patients. For older patients, the amount of exercise should be strictly controlled, avoid intense exercise, and proceed gradually. If the patient appears dizziness, difficulty in breathing or pallor and other symptoms during exercise, the patient should stop exercise immediately and give symptomatic treatment. Through exercise, can strengthen the patient's resistance, prevent complications, promote blood circulation.

4) Diet intervention: make a reasonable diet plan for the patients. Dietary treatment is very important for patients with diabetes. A reasonable diet can help patients maintain stable blood glucose, which is conducive to the treatment of patients. Advise patients to eat more food with high protein, high fiber and easy to digest, eat more fresh vegetables, fruits with less sugar, pasta and wheat, and avoid food with high cholesterol, high sugar, high fat and high calorie, such as animal organs, meat, sweets and fish eggs. Stick to small and many meals, do not overeat, to ensure balanced nutrition. No smoking, no alcohol, no stimulat- 
ing diet, strict control of sugar, salt and calorie intake, control of diet [30]. The patient's blood sugar was controlled by proper exercise, medication and diet.

5) Health education: diabetes-related knowledge, such as pathogenesis, complications and clinical manifestations, should be explained to patients and their families, and the range and normal value of blood glucose fluctuation should be informed so that patients can master knowledge related to diabetes and improve their self-protection ability and prevention and health care ability. Explain treatment considerations to the patient to avoid adverse effects on treatment due to the patient's unhealthy behavior. Instruct patients to conduct blood glucose monitoring, demonstrate how to use the blood glucose detector, let patients monitor blood glucose after meals or on an empty stomach, and enable patients to master the correct method of blood glucose monitoring.

6) Medication intervention: for patients receiving oral treatment with hypoglycemic drugs, patients should be instructed to take medication regularly and according to the amount of medication, not to increase or decrease the amount of medication at will [31]. As elderly people have poor memory and are prone to repeat or forget taking medicine, medical staff should follow up by telephone to understand the treatment of patients, remind family members to supervise patients' taking medicine, divide the medicine according to the dosage, avoid taking the wrong medicine or missing the medicine, and ensure the effect of drug treatment. The patients were treated by insulin injection. Attention should be paid to accurately grasp the dose of insulin injection, select different sites for injection, and sterilize the surrounding skin before injection to avoid the occurrence of infection.

7) Complications prevention: hypoglycemia is a common complication of diabetes, clinical performance for the pale, hungry, flustered, giddy and sweating when the above symptoms in patients with, to take corresponding measures immediately, by eating or drinking sugar water and alleviate the symptoms of low blood sugar, blood can be performed when necessary, check the patients' blood sugar. Elderly diabetic patients are older and prone to infection. Therefore, attention should be paid to infection prevention, skin care of patients, instructing patients to keep warm, pay attention to hygiene, soak their feet in warm water, avoid burns and prevent infection.

\section{Summary}

Limitations of overall nursing management: This study only focuses on the nursing intervention and guidance of diabetic outpatient hospitals. It does not compare hospital-community-individuals in a comprehensive manner, discusses the lack of universality and comprehensiveness, and does not discuss the care of diabetes in other hospitals.

This study should conduct qualitative interviews with patients attending the diabetes care clinic, and further study the needs of patients attending the diabetes care clinic, so as to provide more scientific theoretical basis for improving 
patients' medical experience and promoting the development of the diabetes care clinic.

Assumption: To provide whole-process nursing intervention in the real sense for diabetes patients, it must be a joint intervention between the hospital and community medical health station. Both the hospital and the community should establish the patient's personal file at the same time, and a two-way referral mechanism should be established. When the patient is discharged, the hospital will refer the patient to the community health station, and the nurses in the community will continue to intervene. When patients are seriously ill and need to be hospitalized, they will be referred to the hospital by the community health station, so as to truly share resources, provide continuous medical care services for patients, and benefit the community diabetes patients.

\section{Corresponding Countermeasures and Prospects}

1) Led by the government health administration department, a community professional medical team consisting of more professional doctors, nurses, dietitians and others [32] with guidance on diabetes prevention and control shall be formed to macro-control diabetes, formulate detailed systems and continuous intervention guidance, and relieve the work pressure of medical staff in hospitals; 2) The hospital and community health departments work together to establish personal records for each diabetic patient, where the conditions of the hospital and community are implemented two-way referral, to provide every patient with the whole process of nursing management services; 3 ) Carry out secondary prevention and treatment of diabetes in the community [33], and try to control patients' blood glucose, blood pressure and blood lipid in the community to effectively delay the occurrence of chronic complications; 4) Give play to the role of the computer, establish a sound three-level diabetes prevention network and a perfect diabetes management network throughout the country [34], achieve the sharing of hospital and community resources, and timely provide medical services for patients.

\section{Foundation Project}

Hainan Health Commission Science and Technology Project (No. 20A200002) and Supported by Hainan Natural Science Foundation Project (No. 819MS143).

\section{Conflicts of Interest}

The authors declare no conflicts of interest regarding the publication of this paper.

\section{References}

[1] Xu, M.Y. (2010) Diabetes. 2nd Edition, Shanghai Science and Technology Press, Shanghai, 8. (In Chinese)

[2] Hu, S.L., Liu, G.E., Xu, Z.R., et al. (2008) Current Status of Diabetes Epidemiology 
and Disease Economic Burden in China. Chinese Journal of Health Economics, 27, 5-8.

[3] Wang, C.J. (2014) Prevalence and Prevention of Diabetes. Anhui Medical Science, 35, 1335-1337.

[4] WHO (2015) World Diabetes Report. WHO, Switzerland, 1-5.

[5] Guo, Z. (2015) Study on the Prevalence and Economic Burden of Hypertension and Diabetes Mellitus in Guilin. Chinese Journal of Disease Control, 19, 887-888.

[6] Cheng, J.Y., Zhang, J.H. and Meng, C.L. (2015) Investigation and Analysis on the Status Quo of Diabetes Specialist Nursing Out-Patient Work. Journal of Nursing, 30, 26-29.

[7] Hou, Q. (2016) Current Situation of Global Diabetes Burden. Chinese Journal of Diabetes, 24, 92-94.

[8] Cheng, Z. (2000) The Latest Model of the American Nursing Implementation System-Holistic Nursing Management. Chinese Hospital Management, 20, 6.

[9] Liu, J.H. and Zhang, Y. (2017) Evaluation of the Effect of Outpatient Nursing Intervention on Improving the Quality of Life of Elderly Patients with Diabetes. Journal of Practical Clinical Nursing, 2, 133-134.

[10] Kersbergen, A.L. (1996) Case Management: A Rich History of Coordinating Care to Control Costs. Nursing Outlook, 44, 169-172. https://doi.org/10.1016/S0029-6554(96)80037-6

[11] Wang, J. (2013) Research Review of Case Study Methods. Social Science Management and Review, No. 3, 77-82.

[12] Lin, F. (2015) Practice and Exploration of Zhenjiang City's Health Service System Based on Hierarchical Diagnosis and Treatment. Chinese Journal of Medical Management Science, 5, 19-23.

[13] Fan, L.F., Pan, C.Y., Tian, H., et al. (2001) Establishment and Practice of Whole-Course Diabetes Health Education Model. Chinese Journal of Nursing, 36, 249-252.

[14] Xiao, X.L. (2001) On the Establishment of Health Education and Nursing Clinics in General Hospitals. Modern Nursing, 7, 49-50.

[15] Qian, X.F., Chen, Q.Y., Zhang, S.Z., et al. (2005) Investigation and Research on the Social Needs of Setting up Maternity and Child Care Clinics. Chinese Journal of Nursing, 40, 94-95.

[16] CAN (2008) Advanced Nursing Practice: A National Framework. Canadian Nurses Association, Ottawa, 21-36.

[17] Zhang, L.Y. (2001) The Birth and Development of Senior Nurse Practitioners. Foreign Medical Science: Nursing, 20, 545-547.

[18] He, L.F. and Liao, S.M. (2006) The Establishment of IFL Diagnosis Promotes the Development of Nursing Profession in China. Journal of Nursing Management, 6, 22-24.

[19] Mainie, P.M., Moore, G., Riddell, J.W., et al. (2005) To Examine the Effectiveness of a Hospital-Based Nurse-Led Secondary Prevention Clinic. European Journal of Cardiovascular Nursing, 4, 308-313. https://doi.org/10.1016/j.ejcnurse.2005.04.003

[20] Campbell, J., German, L., Lane, C., et al. (2000) Radiotherapy Outpatient Review: A Nurse-Led Clinic. Clinical Oncology, 12, 104-107. https://doi.org/10.1007/s001740050120

[21] Perrin, A. (2005) Development of a Nurse-Led Ileo-Anal Pouch Clinic. British 
Journal of Nursing, 14, 21-24. https://doi.org/10.12968/bjon.2005.14.Sup4.19739

[22] Ryan, P. (2000) The Benefits of a Nurse-Led Preoperative Assessment Clinic. Nursing Times, 96, 42-43.

[23] Toobert, D.J., Hampson, S.E. and Glasgow, R.E. (2000) The Summary of Diabetes Self-Care Activities Measure: Results from 7 Studies and a Revised Scale. Diabetes Care, 23, 943-950. https://doi.org/10.2337/diacare.23.7.943

[24] Sun, S.N., Zhao, W.G., Dong, Y.Y., et al. (2011) Status Quo and Influencing Factors of Self-Management in Diabetic Patients. Chinese Journal of Nursing, 46, 229-232.

[25] Liao, Z.H., Liang, Y.Q. and Zhou, F.Q. (2000) Quality of Life Is an Important Indicator for Evaluating the Treatment of Diabetes. Chinese Journal of Diabetes, 8 , 304-305.

[26] Zhang, Z.J. (2005) Handbook of Behavioral Medicine Scale. China Medical Electronic Audio-visual Publishing House, Beijing, 114-116.

[27] Zhou, L. and Zhou, Z. (2013) Effect of Outpatient Nursing Intervention on Cognition and Quality of Life of Diabetic Patients. Hebei Medical Science, 4, 162-163.

[28] Jin, Y.X. (2010) Discussion on the Application of Health Education in Elderly Patients with Diabetes. Chinese Community Physicians. Medical Profession, 16, 134-135.

[29] Yang, Z.L. (2012) Evaluation of the Effect of Comprehensive Nursing Intervention on the Quality of Life of Diabetic Patients. China Health and Nutrition, 10, 116-117.

[30] Chen, J.L. (2011) How to Conduct Health Education for Elderly Patients with Diabetes. Medical Information, No. 3, 68-69.

[31] Lang, Y.Z. (2010) Health Education for Elderly Patients with Diabetes. Journal of Baotou Medical College, 6, 112-113.

[32] Tiadelbek, Y. (2009) Evaluation of the Effect of Implementing Diabetes Prevention and Treatment Management by Community General Team. Asia-Pacific Traditional Medicine, 5, 133-135.

[33] Chen, G. (2009) Discussion on Secondary Prevention Measures of Diabetes in Community. Chongqing Medical Science, 38, 1810-1811.

[34] Li, H. and Yang, H.Z. (2006) Current Situation and Problems of Follow-Up for Diabetes Mellitus in China. Journal of Nursing, 13, 33-35. 